International Journal of Business Management and Economic Review

Vol. 4, No. 04; 2021

ISSN: 2581-4664

\title{
WORK-LIFE BALANCE WHEN WORK MEETS HOME
}

\author{
Mrs Richa K Saxena* \\ Assistant Professor - NMIMS Mumbai Campus \\ Dr Shobha A Menon \\ Principal - Valia College of Commerce, Mumbai \\ http://doi.org/10.35409/IJBMER.2021.3293
}

\begin{abstract}
The COVID 19 pandemic forced a reset on work-life balance for employees across all sectors. For a large number of employees, working from home was a new experience. While many of them found WFH good for the work-life balance, others felt the lack of corporate culture while working at home. This study explored the demographic differences in the preferences people experienced towards WFH. The finding clearly exposed the line between gender preferences. While women prefer time with co-workers at the office, men are more likely to spend free time in recreational activities. People perceive that work efficiency is better in the office, and the future intention to WFH is not clearly formed.
\end{abstract}

Keyword: COVID19, Work from home, Work-life Balance, Family, Intention to WFH.

\section{INTRODUCTION}

Work from home is not a new phenomenon in many industries. For example, IT companies have been providing flexible timings and WFH facilities to their employees for years. However, in 2020, when the government imposed the lockdown due to COVID 19 outbreak, WFH was implemented in almost every industry. Indeed, it was a new experience even for the sectors extensively using the internet and online technology. Though this arrangement was convenient to manypeople, it has also led to the disruption in work-life balance.

A report published by Kent University in the UK discussed how WFH had brought changes in the lifestyles of different demographics and life-cycle stages. While most of the people welcomed the WFH, they agreed to have faced challenges of work-life balance. Still, a recent survey conducted by Ernst \& Yong revealed that $54 \%$ of the respondents prefer WFH postpandemic (Bhattacharya, 2021).

In the context of work-life balance, Tietze and Musson (2002) have studied the premise of "work time" and "home-time". The core of work and life organisation lies in the clear distinction of both the timings (Tietze \& Musson, 2002).

This study has the following objectives:

(1) Toexplore the ramification of WFH in an individual's work and home life

(2) To discuss the issues and challenges that emerged due to the new work environment and how it has affected genders and other demographics differently. 
International Journal of Business Management and Economic Review

Vol. 4, No. 04; 2021

ISSN: 2581-4664

(3) To understand the intention to WFH in the future.

\section{LITERATURE REVIEW}

The available literature on WFH primarily covers two different issues. The first category discussed people's preference towards WFH,i.e., the advantages and disadvantages people experienced. The second category discussed the increased burden on women due to the new adjustments needed for WFH.

Purwanto et al. (2020) have identified that WFH helped people overcome several issues like getting up early, grooming, travelling costs, traffic hassles, and non-flexible timings. Further, people are happy to get time for family and recreation and the flexibility to work from anywhere. Disadvantages include the lack of infrastructural facilities at home and added cost of electricity and air conditioning. Research conducted on the employees of an IT firm in Indonesia revealed that WFH has improved performance and has given people an opportunity to devote more time to home (Tiroina \& Mahdani, 2021). A study conducted by Hatayama, Viollaz, \& Winkler (2020), discussed the various amenability for WFH. Jobs requiring meeting people and technical equipment are difficult to manage with online meetings and portable equipment. Internet connection at home is also a significant concern(Hatayama et al., 2020).

A report published by the University of Kent highlighted the positive side of WFH as a preference towards flexible working hours. At the same time, people miss interactions with their colleagues and struggled with a lack of equipment and space (Chung, Seo, Forbes, \& Birkett, 2020). A Price WaterhouseCooper's report on the costs and benefits of WFH outlines that people spent less time in traffic, which resulted in cost savings (Borggreven, 2020b). The negative side of WFH was less collaboration amongst colleagues, low employee engagement, and employee well-being(Borggreven, 2020a).

WFH literature significantly addressed the issue of gender inequality. For example, in a study conducted in Iceland,Hjálmsdóttir and Bjarnadóttir(2021) pointed out that household chores are primarily attributed to females. This is even true in a country that is considered to be a "paradise for women". WFH is difficult for parents with young children as other household responsibilities disrupt work time. In addition, especially women have faced the mental stress of meeting the work requirements while dealing with the young children seeking continuous attention. While women tried to do their best, their mental state has been tired, frustrated and anxious. At the same time, women are also responsible for keeping the mental and emotional well-being of the family.

Further, Power (2020) highlighted how WFH had increased the burden on women. Working women have been a part of the care economy (family needs), paid economy (professional needs) and emotional economy (mental well-being needs). COVID 19 has increased the unpaid part of the responsibilities and overlapped and overshadowed the paid one.

While people struggle with work-life balance issues, many companies are looking at a bright future with WFH. Large technology firms like Google, SAP, Facebook and Accenture have given their employees an option to WFH even with vaccination (ET Bureau, 2021). Amazon has allowed its employees to WFH two days a week(ET Panache, 2021). In addition, more and more 
International Journal of Business Management and Economic Review

Vol. 4, No. 04; 2021

ISSN: 2581-4664

people are refurbishing their homes to make them work-friendly.

\section{RESEARCH METHODOLOGY}

This research aims at exploring the preferences of WFH through exploratory research. First, a qualitative analysis of eight research papers was done using ATLAS.ti software. The codes were generated from the key issues and findings of these research papers. The codes and their respective sentiment density are mentioned in Table 1.

Table 1: Sentiment Analysis Summary

\begin{tabular}{|lllll|}
\hline & Sentiments & & & \\
Codes & Balance & Negative & Neutral & Positive \\
\hline Children & 6 & 17 & 30 & 12 \\
Facilities & 3 & 0 & 5 & 2 \\
Family & 18 & 16 & 24 & 24 \\
Female & 13 & 24 & 41 & 28 \\
Flexible & 8 & 5 & 13 & 9 \\
Gender & 12 & 6 & 24 & 16 \\
Household & 5 & 6 & 10 & 11 \\
Job Satisfaction & 4 & 0 & 4 & 4 \\
Low Job Motivation & 3 & 1 & 3 & 4 \\
Male & 3 & 10 & 18 & 11 \\
Married & 8 & 3 & 6 & 8 \\
Meeting people & 0 & 1 & 1 & 0 \\
Personal life & 1 & 0 & 1 & 1 \\
Privacy and Security & 1 & 0 & 1 & 1 \\
Productivity & 2 & 0 & 2 & 2 \\
Save money & 1 & 0 & 1 & 1 \\
Social Distancing & 3 & 3 & 7 & 1 \\
\hline
\end{tabular}

The visual representation of the same in the Sankey diagram is shown in Figure 1.

Figure 1: Sankey Diagram 
International Journal of Business Management and Economic Review

Vol. 4, No. 04; 2021

ISSN: 2581-4664

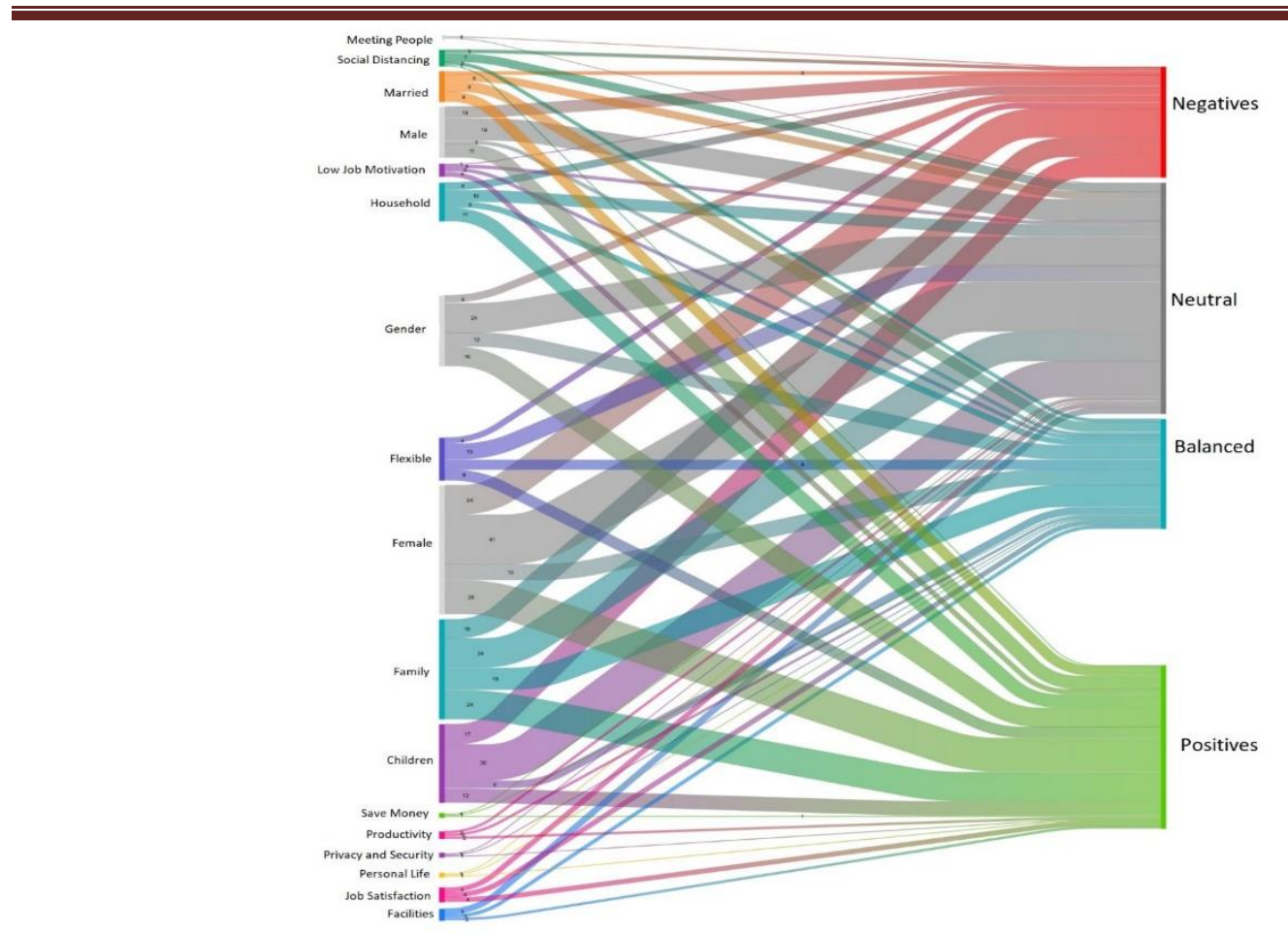

Source: Authors' work using ATLAS.ti

Secondly, open-ended discussions with working professionals, self-employed, services professionals and academicians were conducted.Finally, a list of tenfavourable and 14 unfavourable statements are created,as given in Table 2.

Table 2: Descriptive of Variables

\begin{tabular}{|l|l|l|l|}
\hline Variable & Description & Mean & $\begin{array}{l}\text { Standard } \\
\text { Deviation }\end{array}$ \\
\hline FAV1 & Avoid travelling & 3.45 & 1.199 \\
\hline FAV2 & Availability of freshly cooked home food & 3.36 & 1.300 \\
\hline FAV3 & Flexible work schedule & 3.34 & 1.310 \\
\hline FAV4 & Quality time with family & 3.75 & 1.081 \\
\hline FAV5 & Others at home can help with work & 2.13 & 1.102 \\
\hline FAV6 & Can balance household chores along with work & 3.17 & 1.223 \\
\hline FAV7 & More time to socialise with friends & 2.47 & 1.181 \\
\hline FAV8 & Spare time to pursue hobbies & 3.30 & 1.269 \\
\hline FAV9 & Avoid interactions with co-workers & 2.10 & 1.116 \\
\hline FAV0 & Reduced expenses & 3.71 & 1.129 \\
\hline UNFAV1 & No office pranks and gossip & 2.95 & 1.276 \\
\hline UNFAV2 & No non-work conversations with colleagues & 3.16 & 1.270 \\
\hline UNFAV3 & Not able to supervise subordinates work & 3.04 & 1.149 \\
\hline UNFAV4 & Cannot meet external stakeholders for work & 3.04 & 1.219 \\
\hline UNFAV5 & The office environment is good for cross learnings & 3.99 & 1.055 \\
\hline
\end{tabular}




\section{International Journal of Business Management and Economic Review}

Vol. 4, No. 04; 2021

ISSN: 2581-4664

\begin{tabular}{|l|l|l|l|}
\hline \hline UNFAV6 & Not able to meet and network with people & 3.64 & 1.179 \\
\hline UNFAV7 & Affects physical fitness & 3.66 & 1.150 \\
\hline UNFAV8 & No socialising and meeting friends & 3.47 & 1.218 \\
\hline UNFAV9 & $\begin{array}{l}\text { No snacks/tea/coffee otherwise provided by the } \\
\text { employer }\end{array}$ & 2.19 & 1.063 \\
\hline UNFAV10 & $\begin{array}{l}\text { No recreational facilities otherwise provided by the } \\
\text { employer }\end{array}$ & 2.23 & 1.068 \\
\hline UNFAV11 & Frequently distracted by background noises & 3.33 & 1.261 \\
\hline UNFAV12 & Poor internet stability/network issues & 3.39 & 1.309 \\
\hline UNFAV13 & More tiring due to longer working hours & 3.54 & 1.236 \\
\hline UNFAV14 & Family is around all the time & 2.49 & 1.142 \\
\hline
\end{tabular}

Further, the literature review and the interview process revealed that these differences were not equally relevant for different demographics. Therefore, statistical analysis was conducted to explore the differences amongst genders, marital status, age groups, people with or without children, people living with the elderly, type of residence, family type, and sectors. Further, a combination of these factors was also explored to understand their effect.

\section{DATA COLLECTION}

The responses were collected on favourable and unfavourable statements using a 5 point Likert scalewhere 1-strongly disagree, 2-disagree, 3-neutral, 4-agree and 5-strongly agree(Vagias \& Wade, 2006). The respondents were also asked for their perception of efficiency when working from the office against working at home. Finally, they were asked about their intention to work from home. The data was collected using an online questionnaire from July 2020 to October 2020. The details of the responses are given in Table 3.

The preferences were tested for normality using Kolmogorov-Smirnov and Shapiro-Wilk tests. Both tests showed $\mathrm{p}<0.001$ for all the variables. Therefore, all variables are significantly nonnormal. Thus, for the hypothesis testing, non-parametric tests were used.

Table 3: Demographic and other Variables

\begin{tabular}{|c|c|c|c|}
\hline \multicolumn{2}{|l|}{ Demographic } & Frequency & Percent \\
\hline \multirow[t]{2}{*}{ Gender } & Male & 75 & 56.0 \\
\hline & Female & 59 & 44.0 \\
\hline \multirow{4}{*}{ Age } & $21-30$ & 83 & 61.9 \\
\hline & $31-40$ & 27 & 20.1 \\
\hline & $41-50$ & 20 & 14.9 \\
\hline & above 50 & 4 & 3.0 \\
\hline \multirow{2}{*}{ Marital Status } & Single & 73 & 54.5 \\
\hline & Married & 61 & 45.5 \\
\hline \multirow{2}{*}{ Children } & None & 92 & 68.7 \\
\hline & Yes & 42 & 31.3 \\
\hline
\end{tabular}


International Journal of Business Management and Economic Review

Vol. 4, No. 04; 2021

ISSN: 2581-4664

\begin{tabular}{|l|l|l|l|}
\hline \hline \multirow{5}{*}{ Elderly } & None & 69 & 51.5 \\
& Yes & 65 & 48.5 \\
\hline \multirow{5}{*}{ Fesidence } & 1RK & 30 & 22.4 \\
& 1BHK & 40 & 29.9 \\
& 2BHK & 42 & 31.3 \\
& 3BHK & 22 & 16.4 \\
\hline \multirow{5}{*}{ Sector } & Alone & 9 & 6.7 \\
& with friends & 2 & 1.5 \\
& nuclear family & 98 & 73.1 \\
\hline \multirow{5}{*}{ Efficiency } & extended family & 25 & 18.7 \\
\hline \multirow{3}{*}{ Intentions } & Corporate & 91 & 67.9 \\
& Education & 28 & 20.9 \\
& Self Employed & 8 & 6.0 \\
& Government and Healthcare & 7 & 5.2 \\
\hline & Working from Office & 78 & 58.2 \\
& Working from home & 16 & 11.9 \\
& No difference & 40 & 29.9 \\
\hline
\end{tabular}

\section{DATA ANALYSIS}

First, the descriptive analysis was conducted using means and standard deviations. From Table 2, it is evident that for the favourable statements, the highest agreement is with "quality time with family" (mean=3.75), followed by "reduced expenses" (mean=3.71). These statements also have the smallest variation amongst respondents. The least agreement is with the statements "avoid interaction with co-workers" (mean=2.10) and "others at home can help with work" (mean=2.13)."Flexible hours" and "availability of freshly cooked home food" have a relatively high agreement but also has the highest variation. Figure 2 shows the visual representation of the level of agreement for favourable statements.

For unfavourable statements, the highest agreement is for "office environment is good for cross learnings" (mean=3.99). The second highest agreement is for the statement "affects physical fitness" (mean=3.66). Both the statements also have small variations. The least agreement for unfavourable statements is for "no snack/tea/coffee" (mean=2.19) and "no recreational facilities" (2.23) otherwise provided by the employer. Figure 3 shows the visual representation of the level of agreement with the unfavourable statements. 
International Journal of Business Management and Economic Review

Vol. 4, No. 04; 2021

ISSN: 2581-4664

Figure 2: Level of Agreement for Favourable Statements

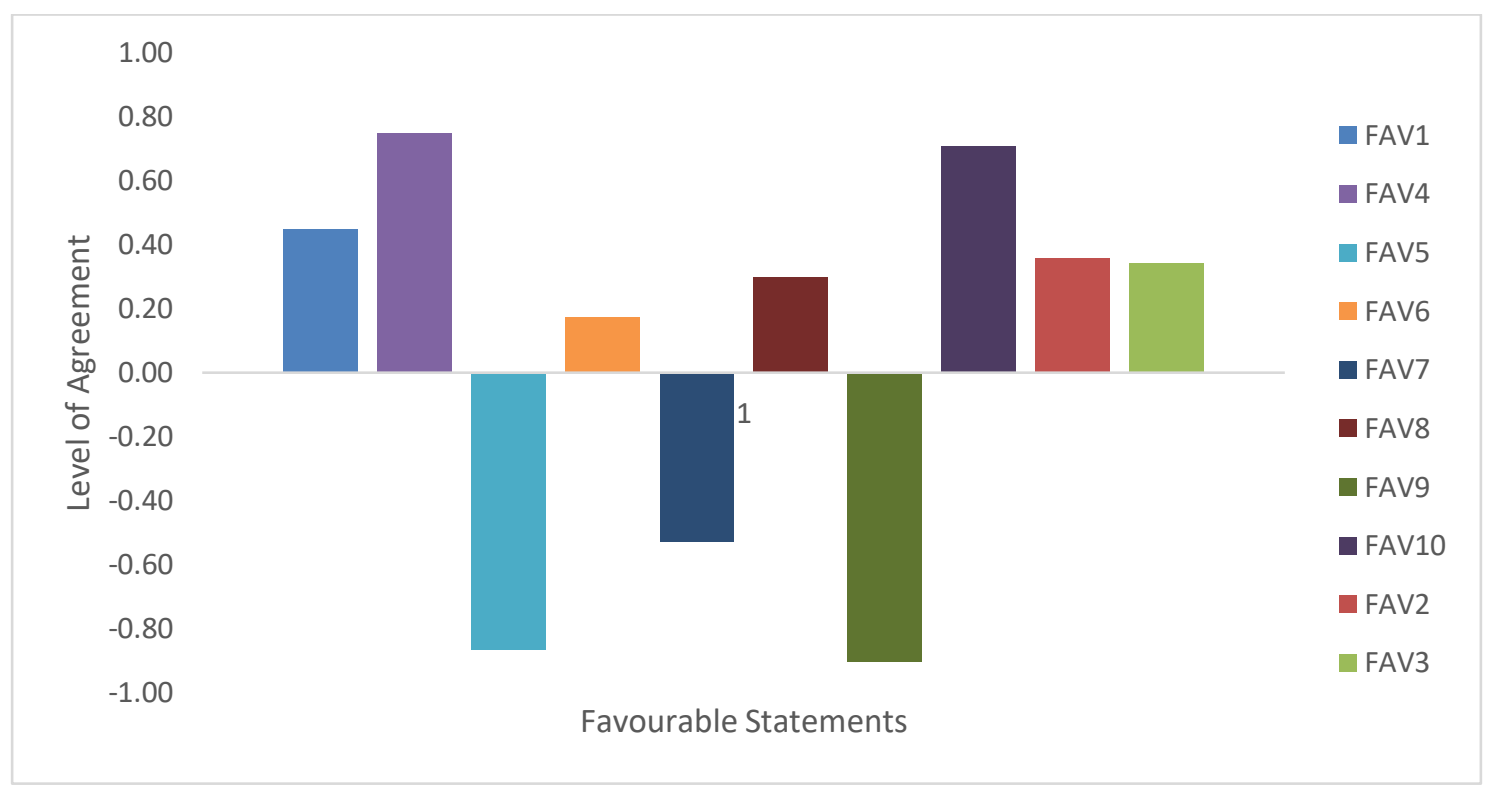

Source: Authors' work using MS Excel

Figure 3: Level of Agreement for Unfavourable Statements

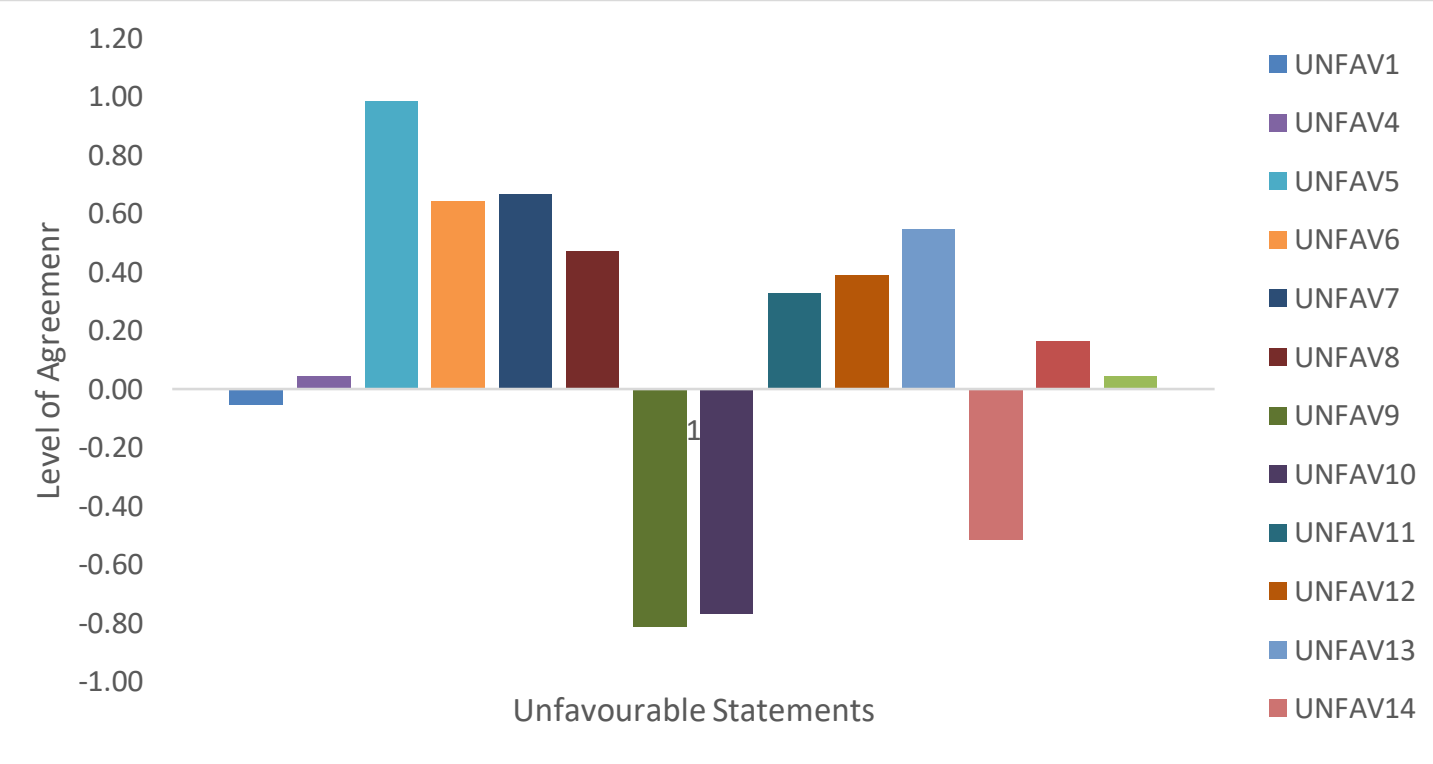

Source: Authors' work using MS Excel

Next, the group difference analysis was conducted. Mann-Whitney test for 2-independent samples was used to test the difference between the opinion of male and female respondents. As 


\section{International Journal of Business Management and Economic Review}

Vol. 4, No. 04; 2021

ISSN: 2581-4664

shown in Table 4, four favourable statements and three unfavourable statements are significantly different for male and female respondents at $\mathrm{p}<0.05$.

Table 4: Test Statistics for Gender Differences

\begin{tabular}{|c|c|c|c|c|c|c|c|}
\hline \multirow[b]{2}{*}{ Statements } & \multirow{2}{*}{$\begin{array}{l}\text { Mann- } \\
\text { Whitney } \\
\text { U }\end{array}$} & \multirow{2}{*}{$\begin{array}{l}\text { Wilcoxon } \\
\text { W }\end{array}$} & \multirow[b]{2}{*}{ Z } & \multirow{2}{*}{$\begin{array}{l}\text { Asymp. } \\
\text { Sig. (2- } \\
\text { tailed) }\end{array}$} & \multicolumn{2}{|c|}{ Mean Rank } & \multirow[b]{2}{*}{ Inference } \\
\hline & & & & & Male & female & \\
\hline FAV2 & 1731.500 & 3501.500 & -2.216 & 0.027 & 73.91 & 59.350 & \multirow{4}{*}{$\begin{array}{l}\text { male respondents } \\
\text { agreed more than } \\
\text { female respondents }\end{array}$} \\
\hline FAV4 & 1743.500 & 3513.500 & -2.190 & 0.029 & 73.75 & 59.550 & \\
\hline FAV7 & 1687.500 & 3457.500 & -2.432 & 0.015 & 74.50 & 58.600 & \\
\hline FAV8 & 1896.000 & 3666.000 & -1.476 & 0.140 & 71.72 & 62.140 & \\
\hline UNFAV5 & 1680.500 & 4530.500 & -2.565 & 0.010 & 60.41 & 76.520 & \multirow{3}{*}{$\begin{array}{l}\text { female respondents } \\
\text { agree more than male } \\
\text { respondents }\end{array}$} \\
\hline UNFAV12 & 1644.500 & 4494.500 & -2.621 & 0.009 & 59.93 & 77.130 & \\
\hline UNFAV14 & 1540.000 & 4390.000 & -3.119 & 0.002 & 58.53 & 78.900 & \\
\hline
\end{tabular}

a. Grouping Variable: Gender

Next, the test was run to identify significant differences in the responses of single and married respondents. The significant statements are given in Table 5.

Table 5: Test Statistics for differences due to Martial Status

\begin{tabular}{|c|c|c|c|c|c|c|c|}
\hline \multirow{2}{*}{ Statement } & \multirow{2}{*}{$\begin{array}{l}\text { Mann- } \\
\text { Whitney } \\
\text { U }\end{array}$} & \multirow{2}{*}{$\begin{array}{l}\text { Wilcoxo } \\
n \mathrm{~W}\end{array}$} & \multirow[b]{2}{*}{ Z } & \multirow{2}{*}{$\begin{array}{l}\text { Asymp. } \\
\text { Sig. (2- } \\
\text { tailed) }\end{array}$} & \multicolumn{2}{|c|}{ Mean Rank } & \multirow{2}{*}{ Inference } \\
\hline & & & & & Male & female & \\
\hline FAV2 & 1696.5 & 3526.5 & -2.407 & 0.016 & 74.57 & 58.78 & \multirow{4}{*}{$\begin{array}{l}\text { single respondents } \\
\text { agree more than } \\
\text { married respondents }\end{array}$} \\
\hline FAV7 & 1608.5 & 3438.5 & -2.828 & 0.005 & 75.76 & 57.31 & \\
\hline FAV10 & 1683.5 & 3513.5 & -2.501 & 0.012 & 74.75 & 58.56 & \\
\hline UNFAV1 & 1951.5 & 3781.5 & -1.230 & 0.218 & 71.13 & 63.03 & \\
\hline
\end{tabular}

a. Grouping Variable: Marital Status

Since the data consists of four age groups, K-means sample non-parametric test is used to test the difference amongst age groups. Table 6 represents the statistics. Only one statement showed a significant difference amongst the respondents. 


\section{International Journal of Business Management and Economic Review}

Vol. 4, No. 04; 2021

ISSN: 2581-4664

\section{Table 6: Test Statistics for Age Groups}

\begin{tabular}{|c|c|c|c|c|c|c|c|c|}
\hline \multirow{2}{*}{ Statement } & \multirow{2}{*}{$\begin{array}{l}\text { Kruskal- } \\
\text { Wallis H }\end{array}$} & \multirow{2}{*}{$\mathrm{df}$} & \multirow{2}{*}{$\begin{array}{l}\text { Asymp. } \\
\text { Sig. }\end{array}$} & \multicolumn{4}{|c|}{ Mean Rank } & \multirow{2}{*}{ Inference } \\
\hline & & & & $\begin{array}{l}21-30 \\
\text { years }\end{array}$ & $\begin{array}{l}31-40 \\
\text { years }\end{array}$ & $\begin{array}{l}41-50 \\
\text { years }\end{array}$ & $\begin{array}{l}\text { Above } \\
50 \text { years }\end{array}$ & \\
\hline FAV10 & 11.8095 & 3 & 0.008 & 75.14 & 51.5 & 64 & 34.38 & $\begin{array}{l}21-30 \text { agreed more, } \\
\text { and above } 50 \text { agreed } \\
\text { less than the rest }\end{array}$ \\
\hline
\end{tabular}

a. Kruskal Wallis Test

b. Grouping Variable: Age Groups

K-means sample is also used for differences in four categories of types of residents. Table 7 represents the test statistics for the same.

Table 7: Test Statistics for differences in residential status

\begin{tabular}{|c|c|c|c|c|c|c|c|c|}
\hline \multirow[b]{2}{*}{ Statements } & \multirow{2}{*}{$\begin{array}{l}\text { Kruskal- } \\
\text { Wallis } \\
\text { H }\end{array}$} & \multirow[b]{2}{*}{$\mathrm{df}$} & \multirow{2}{*}{$\begin{array}{l}\text { Asymp. } \\
\text { Sig. }\end{array}$} & \multicolumn{4}{|c|}{ Mean Rank } & \multirow[b]{2}{*}{ Inference } \\
\hline & & & & $1 \mathrm{RK}$ & 1BHK & $2 \mathrm{BHK}$ & 3BHK & \\
\hline FAV5 & 8.383 & 3 & 0.039 & 76.07 & 69.05 & 70.36 & 47.55 & \multirow{2}{*}{$\begin{array}{l}\text { People living in } 3 \mathrm{BHK} \\
\text { agree less }\end{array}$} \\
\hline FAV9 & 9.158 & 3 & 0.027 & 74.83 & 72.23 & 68.81 & 46.41 & \\
\hline UNFAV5 & 9.458 & 3 & 0.024 & 66.02 & 66.21 & 69.07 & 87.95 & \multirow{2}{*}{$\begin{array}{l}\text { People living in } 3 \mathrm{BHK} \\
\text { agree more }\end{array}$} \\
\hline UNFAV6 & 11.364 & 3 & 0.010 & 59.73 & 66.19 & 61.8 & 91.36 & \\
\hline
\end{tabular}

a. Kruskal Wallis Test

b. Grouping Variable: RESIDENCE

Next, the analysis was done, creating different groups of males and female respondents, and 2sample mean the test was run to test the differences based on marital status. The results are displayed in Table 8.

Table 8: Test Statistics for Marital Status * Gender

\begin{tabular}{|l|l|l|l|l|l|l|l|}
\hline Statements & $\begin{array}{l}\text { Mean } \\
\text { Rank }\end{array}$ & $\begin{array}{l}\text { Wilcoxon } \\
\text { W }\end{array}$ & $Z$ & $\begin{array}{l}\text { Asymp. } \\
\text { Sig. (2- } \\
\text { tailed) }\end{array}$ & $\begin{array}{l}\text { Mean Rank } \\
\begin{array}{l}\text { Single } \\
\text { male }\end{array}\end{array}$ & $\begin{array}{l}\text { Married } \\
\text { male }\end{array}$ & Inferences \\
\hline FAV10 & 441.000 & 1107.000 & -2.924 & 0.003 & 44.69 & 30.750 & $\begin{array}{l}\text { single male respondents } \\
\text { agree more than married } \\
\text { male respondents }\end{array}$ \\
\hline
\end{tabular}

a. GEN = 1 Male

b. Grouping Variable: MS

\begin{tabular}{|l|l|l|l|l|l|l|l|}
\hline Statements & $\begin{array}{l}\text { Mann- } \\
\text { Whitne } \\
\text { y U }\end{array}$ & $\begin{array}{l}\text { Wilcoxon } \\
\text { W }\end{array}$ & Z & $\begin{array}{l}\text { Asymp. } \\
\text { Sig. (2- } \\
\text { tailed) }\end{array}$ & $\begin{array}{l}\text { Single } \\
\text { female }\end{array}$ & $\begin{array}{l}\text { Married } \\
\text { female }\end{array}$ & Inferences \\
\hline
\end{tabular}




\section{International Journal of Business Management and Economic Review}

Vol. 4, No. 04; 2021

ISSN: 2581-4664

\begin{tabular}{|c|c|c|c|c|c|c|c|}
\hline FAV7 & 265.500 & 565.500 & -2.488 & 0.013 & 34.41 & 23.560 & \multirow{3}{*}{$\begin{array}{l}\text { single female } \\
\text { respondents agree more } \\
\text { than married female } \\
\text { respondents }\end{array}$} \\
\hline UNFAV10 & 291.000 & 591.000 & -2.074 & 0.038 & 33.69 & 24.630 & \\
\hline UNFAV12 & 273.500 & 573.500 & -2.360 & 0.018 & 34.19 & 23.900 & \\
\hline
\end{tabular}

a. $\mathrm{GEN}=2$ Female

b. Grouping Variable: MS

Also, the analysis was done after grouping the data into marital statuses. The differences in male and female responses were identified. The significant differences are given in Table 9.

Table 9: Test Statistics for Gender * Marital Status

\begin{tabular}{|l|l|l|l|l|l|l|l|}
\hline Statements & $\begin{array}{l}\text { Mann- } \\
\text { Whitney } \\
\text { U }\end{array}$ & $\begin{array}{l}\text { Wilcoxon } \\
\text { W }\end{array}$ & $Z$ & $\begin{array}{l}\text { Asymp. } \\
\text { Sig. (2- } \\
\text { tailed) }\end{array}$ & $\begin{array}{l}\text { Single } \\
\text { male }\end{array}$ & $\begin{array}{l}\text { Single } \\
\text { Female }\end{array}$ & Inferences \\
\hline UNFAV12 & 390.000 & 1170.000 & -3.262 & 0.001 & 30 & 45.86 & $\begin{array}{l}\text { single female } \\
\text { respondents agree } \\
\text { more than single male } \\
\text { respondents }\end{array}$ \\
\hline UNFAV14 & 490.500 & 1270.500 & -2.142 & 0.032 & 32.58 & 42.99 \\
\hline
\end{tabular}

a. $\mathrm{MS}=1$ Single

b. Grouping Variable: GEN

\begin{tabular}{|c|c|c|c|c|c|c|c|}
\hline & & & & & Mean $\mathrm{R}$ & ink & \\
\hline Statements & $\begin{array}{l}\text { Mann- } \\
\text { Whitney } \\
\text { U }\end{array}$ & $\begin{array}{l}\text { Wilcoxon } \\
\text { W }\end{array}$ & $\mathrm{Z}$ & $\begin{array}{l}\text { Asymp. } \\
\text { Sig. (2- } \\
\text { tailed) }\end{array}$ & $\begin{array}{l}\text { Marrie } \\
\text { d male }\end{array}$ & $\begin{array}{l}\text { Married } \\
\text { female }\end{array}$ & Inferences \\
\hline FAV7 & 294.500 & 594.500 & -2.168 & 0.030 & 34.32 & 24.77 & married male \\
\hline FAV8 & 292.500 & 592.500 & -2.210 & 0.027 & 34.38 & 24.69 & $\begin{array}{l}\text { respondents agree } \\
\text { more than married }\end{array}$ \\
\hline UNFAV3 & 290.500 & 590.500 & -2.220 & 0.026 & 34.43 & 24.6 & female respondents \\
\hline
\end{tabular}

a. $\mathrm{MS}=2$ Married

b. Grouping Variable: GEN

There is no significant difference identified in people's responses with children or no children or living or not living with the elderly. Next, the data for single and married male and female respondents living with or without elderly was analysed. The significant differences are given in Table 10.

Table 10 Test Statistics for Marital Status*Gender*Having Elderly or not

\begin{tabular}{|c|c|c|c|c|c|c|c|c|}
\hline \multirow[b]{2}{*}{ Statements } & \multirow[b]{2}{*}{$\begin{array}{l}\text { Mann- } \\
\text { Whitney } \\
\text { U }\end{array}$} & \multirow[b]{2}{*}{$\begin{array}{l}\text { Wilcoxon } \\
\text { W }\end{array}$} & \multirow[b]{2}{*}{ Z } & \multirow[b]{2}{*}{$\begin{array}{l}\text { Asymp. } \\
\text { Sig. (2- } \\
\text { tailed) }\end{array}$} & \multirow{2}{*}{$\begin{array}{l}\text { Exact } \\
\text { Sig. } \\
{[2 *(1-} \\
\text { tailed } \\
\text { Sig.)] }\end{array}$} & \multicolumn{2}{|c|}{ Mean Rank } & \multirow[b]{2}{*}{ Inferences } \\
\hline & & & & & & $\begin{array}{l}\text { No } \\
\text { Elderly }\end{array}$ & $\begin{array}{l}\text { With } \\
\text { Elderly }\end{array}$ & \\
\hline
\end{tabular}


International Journal of Business Management and Economic Review

Vol. 4, No. 04; 2021

ISSN: 2581-4664

\begin{tabular}{|c|c|c|c|c|c|c|c|c|}
\hline FAV2 & 108.500 & 339.500 & -2.387 & 0.017 & 0.022 & 16.17 & 24.47 & \multirow{4}{*}{$\begin{array}{l}\text { Respondents } \\
\text { living with the } \\
\text { elderly agree } \\
\text { more than not } \\
\text { living with } \\
\text { elderly }\end{array}$} \\
\hline FAV4 & 114.000 & 345.000 & -2.212 & 0.027 & 0.035 & 16.43 & 24.17 & \\
\hline FAV7 & & 25.0 & -2.7 & 0.0 & 0.00 & 15. & 5. & \\
\hline UNFAV8 & 118.500 & 280500 & -2.061 & 0030 & 0.046 & 23.36 & 16.08 & \\
\hline
\end{tabular}

a. MS $=1$ Single, GEN = 1 Male

b. Grouping Variable: ELDERLY

\begin{tabular}{|c|c|c|c|c|c|c|c|c|}
\hline FAV8 & 79 & 250 & -2.529 & 0.011 & 0.014 & 22.35 & 13.89 & $\begin{array}{l}\text { Respondents } \\
\text { living with the } \\
\text { elderly agree } \\
\text { more than not } \\
\text { living with } \\
\text { elderly }\end{array}$ \\
\hline
\end{tabular}

a MS = 1 Single, GEN = 2 Female

b Grouping Variable: ELDERLY

\begin{tabular}{|l|l|l|l|l|l|l|l|l|}
\hline UNFAV1 & 34 & 79 & -2.071 & 0.038 & 0.048 & 8.78 & 14.17 & $\begin{array}{l}\text { Respondents not } \\
\text { living with the } \\
\text { elderly agree } \\
\text { more than not } \\
\text { living with } \\
\text { elderly }\end{array}$ \\
\hline UNFAV7 & 32 & 77 & -2.214 & 0.027 & 0.035 & 8.56 & 14.87 \\
\hline
\end{tabular}

a MS = 2 Married, GEN = 2 Female

b Grouping Variable: ELDERLY

Next is the people's response to the statement compared between people living alone, with family or friends. Test statistics are given in Table 11

Table 11: Test Statistics for Type of Family

\begin{tabular}{|c|c|c|c|c|c|c|c|c|}
\hline \multirow[b]{2}{*}{ Statements } & \multirow{2}{*}{$\begin{array}{l}\text { Kruskal- } \\
\text { Wallis } \\
\text { H }\end{array}$} & \multirow[b]{2}{*}{ df } & \multirow{2}{*}{$\begin{array}{l}\text { Asymp. } \\
\text { Sig. }\end{array}$} & \multicolumn{4}{|c|}{ Mean Rank } & \multirow[b]{2}{*}{ Inference } \\
\hline & & & & Alone & $\begin{array}{l}\text { with } \\
\text { friends }\end{array}$ & $\begin{array}{l}\text { Nuclear } \\
\text { Family }\end{array}$ & $\begin{array}{l}\text { Extended } \\
\text { Family }\end{array}$ & \\
\hline FAV6 & 11.1389 & 3 & 0.011 & 100.5 & 18.75 & 64.53 & 71.16 & \multirow{2}{*}{$\begin{array}{l}\text { people living with } \\
\text { friends agree least } \\
\text { with the statement }\end{array}$} \\
\hline UNFAV13 & 11.8837 & 3 & 0.0078 & 28.39 & 98 & 70.85 & 66 & \\
\hline
\end{tabular}

a. Kruskal Wallis Test

b. Grouping Variable: FAMILY

Sector-wise comparison and the results are reflected in Table 12: 
International Journal of Business Management and Economic Review

Vol. 4, No. 04; 2021

ISSN: 2581-4664

\section{Table 12: Test Statistics for Sectors}

\begin{tabular}{|l|l|l|l|l|l|l|l|l|}
\hline Statements & $\begin{array}{l}\text { Kruskal } \\
\text {-Wallis } \\
\text { H }\end{array}$ & df & $\begin{array}{l}\text { Asymp. } \\
\text { Sig. }\end{array}$ & Corporate & $\begin{array}{l}\text { Academi } \\
\text { cs }\end{array}$ & $\begin{array}{l}\text { Self- } \\
\text { employe } \\
\mathrm{d}\end{array}$ & $\begin{array}{l}\text { Public } \\
\text { Sector }\end{array}$ & Inference \\
\hline FAV5 & 10.650 & 3 & 0.014 & 61.42 & 87.23 & 63.94 & 71.64 & $\begin{array}{l}\text { Academicians } \\
\text { agree more than } \\
\text { others }\end{array}$ \\
\hline FAV6 & 8.847 & 3 & 0.031 & 61.68 & 79.04 & 94.19 & 66.5 & $\begin{array}{l}\text { Self-employed } \\
\text { and } \\
\text { academicians } \\
\text { agree more than } \\
\text { others }\end{array}$ \\
\hline FAV10 & 8.919 & 3 & 0.030 & 71.38 & 49.98 & 68.44 & 86.07 & $\begin{array}{l}\text { Public sector } \\
\text { employees agree } \\
\text { most, } \\
\text { Academicians } \\
\text { agree least }\end{array}$ \\
\hline UNFAV13 & 21.234 & 3 & 0.000 & 76.27 & 48.5 & 27.94 & 74.71 & $\begin{array}{l}\text { Public and } \\
\text { private sector } \\
\text { agree maximum, } \\
\text { self-employed } \\
\text { agreed least }\end{array}$ \\
\hline
\end{tabular}

a. Kruskal Wallis Test

b. Grouping Variable: SEC

Finally, the intention of the people to WFH in future was assessed. Figure 4 shows the visual representation of people's perception of the efficiency in work at home vs office. Clearly, people felt that efficiencies are more at the office. Given that, people are not able to form the intention to WFH. Figure 5 provides the visual representation of the intention to WFH. 
International Journal of Business Management and Economic Review

Vol. 4, No. 04; 2021

ISSN: 2581-4664

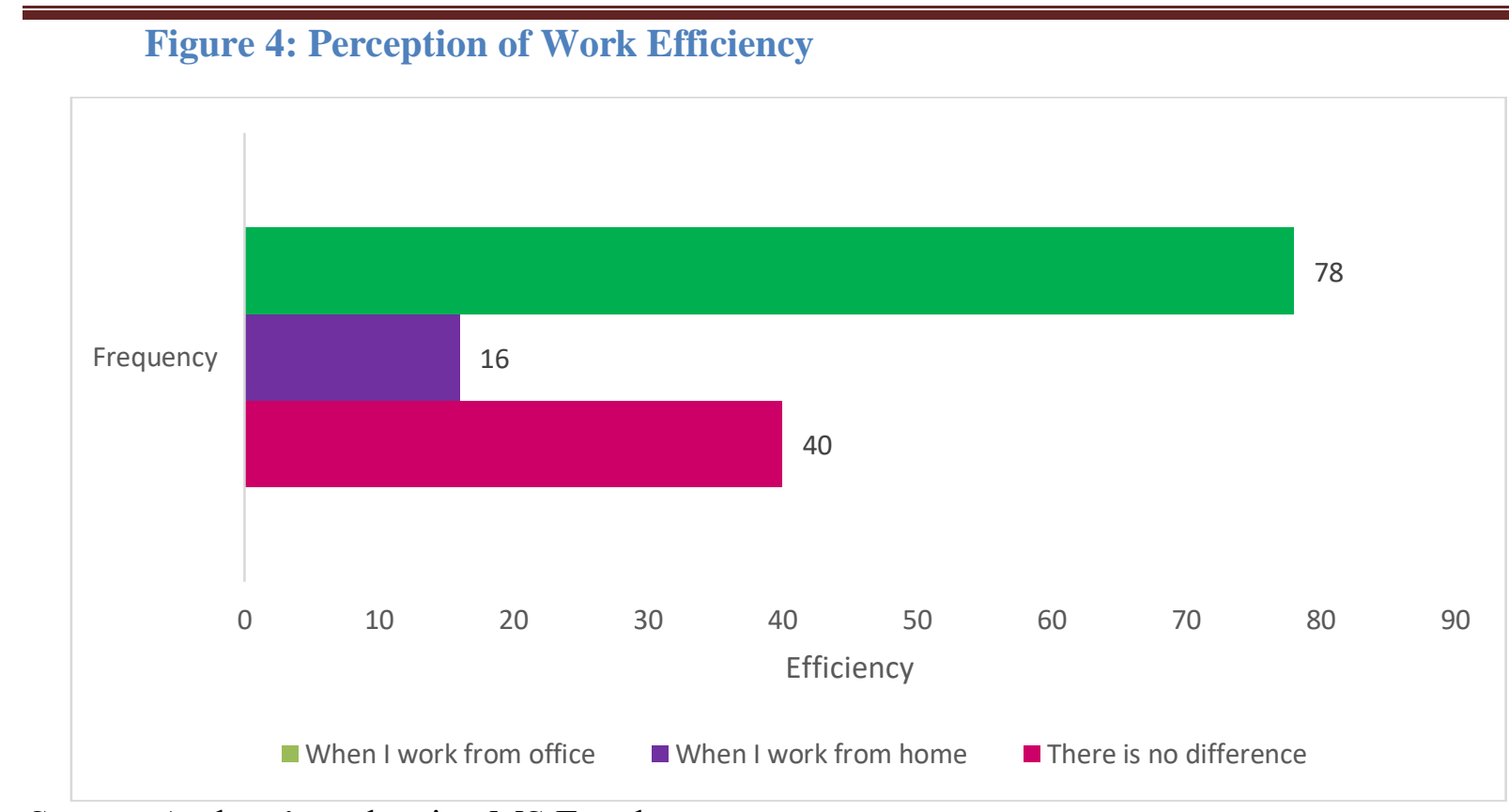

Source: Authors' work using MS Excel

Figure 5: Intention to WFH

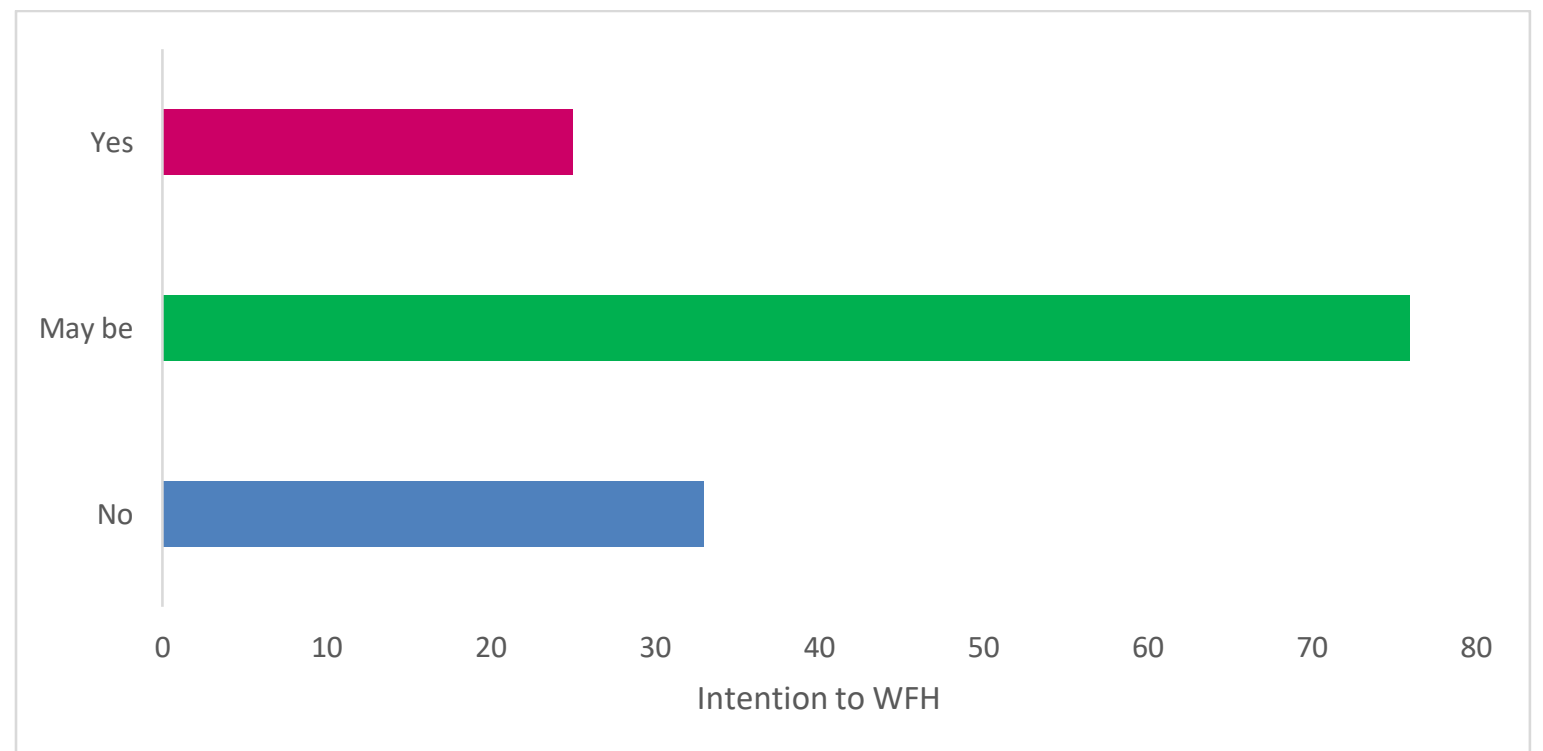

Source: Authors' work using MS Excel

\section{FINDINGS AND DISCUSSIONS}

The first discussion is about the difference of opinion between genders. Male respondents are more inclined towards favourable statements. They get freshly home-cooked food, have time to spend with family and friends, and pursue hobbies. Married males appear to like the latter two more than married females. On the other hand, female respondents majorly agreed with unfavourable statements being surrounded by family during work hours and are affected by poor 


\section{International Journal of Business Management and Economic Review}

Vol. 4, No. 04; 2021

ISSN: 2581-4664

internet.

Moreover, female respondents missed the learning environment of the office. Evidently, these differences are due to work plus home responsibilities for married female respondents even during work hours. As a result, married female respondents did not get much time to socialise or pursue anything else. However, the single female does agree that they got more time to interact with friends than married women. Next, single male and female respondents liked getting freshly cooked food, talking to friends, and reduced expenses than married men and women. However, they also missed office gossips more than married ones. Also, single men liked the reduction in expenses more than married men.

Single female respondents seem to miss office recreation and internet speed than married women. Between single men and women, differences occur in two unfavourable statements internet facilities and being surrounded by family all the time. Evidently, female respondents are affected more than male respondents. Married men disliked missing subordinates more than married women.

Age groups wise analysis showed aged 21-30 years are most relieved due to reduction in expenses, and 50 and above are least affected by such reduction. People living in big houses (3BHK) seems to be preferring the office environment. They agreed more that they do not get to explore cross-learning and cannot meet or network. Instead, they agree less with the statement that people at home can be of any help. They preferred office interactions.

There are no significant differences found in the liking or disliking of people having or not having children. However, single males living with the elderly agreed more on the availability of freshly cooked home food and spare time for family and friends. On the other hand, single female respondents liked that they get to pursue their hobbies due to the presence of the elderly in the family. Also, single men not living with the elderly disliked not being able to socialise due to lockdown. There is no significant difference found in the opinion of married men living or not living with the elderly. However, married females not living with the elderly appear to be missing office pranks. They also feel that their physical fitness is affected.

Next, people living with friends cannot balance work and household chores. On the contrary, people living alone find work more tiring due to long hours. The academicians agreed that they could get help from others and balance work and household chores. However, they do not agree to have saved expenses. Self-employed also agreed to manage work-life better than other sectors and disagree with having put more working hours. Public sector employees appear to be saving expenses but are also putting in more working hours. The employees in the private sector also feel that they are putting more hours into work.

Though people believe that it is more efficient to work from the office, they are not sure if they want to go to the office in the future. Other than the advantages of WFH, safety from the pandemic also can be the reason for the same.

The findings of this research direct towards a mixed arrangement that can create a balance between work efficiencies and comfort of home, savings and overall well-being, post-pandemic. Employers should consider providing flexibility to employees to WFH. In the cases where physical meetings are necessary or specific equipment to be used, employees can work from the office.

\section{CONCLUSIONS AND LIMITATIONS}

The findings of the paper revealed that the WFH has significantly distorted the work-life 


\section{International Journal of Business Management and Economic Review}

Vol. 4, No. 04; 2021

ISSN: 2581-4664

balance. The issues related to gender disparities surfaced even with this small sample. While men utilise more spare time in recreation, women have to prioritise their household liabilities, which is difficult without support. Private and public sector employees see an increase in overall working hours. This research could not find any implications due to the responsibilities of young children. Also, there is no significant difference in the preference or intention to continue to WFH across demographics.

\section{REFERENCES}

Bhattacharya, R. (2021). Even after Covid, most would prefer flexibility in work: EY Survey The Economic Times. Economic Times. Retrieved from https://economictimes.indiatimes.com/jobs/post-covid-most-would-prefer-flexibility-inwork/articleshow/83126513.cms

Borggreven, M. (2020a). The costs and benefits of working from home, Part II: The impact of working from home on innovation, people engagement and well-being. PricewaterhouseCoopers $\quad(P w C) \quad B V \quad(K v K$ 34180289), 1-11. Retrieved from https://www.pwc.nl/nl/actueel-publicaties/assets/pdfs/pwc-the-costs-and-benefits-ofworking-from-home.pdf

Borggreven, M. (2020b). The costs and benefits of working from home. PricewaterhouseCoopers $(P w C) \quad B V \quad(K v K$ 34180289), 1-11. Retrieved from https://www.pwc.nl/nl/actueel-publicaties/assets/pdfs/pwc-the-costs-and-benefits-ofworking-from-home.pdf

Chung, H., Seo, H., Forbes, S., \& Birkett, O. (2020). Working From Home During The Covid-19 Lockdown: Changing Preferences And The Future of Work. University of Birmingham and University of Kent.

ET Bureau, S. K. (2021, June 13). Increasing number of companies looking to shift permanently to flexible, fully remote work model - The Economic Times. Economic Times. Retrieved from https://economictimes.indiatimes.com/industry/services/property-/cstruction/increasing-number-of-companies-looking-to-shift-permanently-to-flexible-fullyremote-work-model/articleshow/83476977.cms

ET Panache, A. (2021, June 11). Amazon allows employees to work from home 2 days a week The Economic Times. Retrieved June 15, 2021, from https://economictimes.indiatimes.com/magazines/panache/amazon-allows-employees-towork-from-home-2-days-a-week/articleshow/83426358.cms

Hatayama, M., Viollaz, M., \& Winkler, H. (2020). Jobs' Amenability to Working from Home: Evidence from Skills Surveys for 53 Countries. Jobs' Amenability to Working from Home: Evidence from Skills Surveys for 53 Countries. https://doi.org/10.1596/1813-9450-9241

Hjálmsdóttir, A., \& Bjarnadóttir, V. S. (2021). "I have turned into a foreman here at home": Families and work-life balance in times of COVID-19 in a gender equality paradise. Gender, Work \& Organisation. https://doi.org/10.1111/gwao.12552

Power, K. (2020). The COVID-19 pandemic has increased the care burden of women and families. Sustainability: Science, Practice, and Policy, 16(1), 67-73. https://doi.org/10.1080/15487733.2020.1776561

Tietze, S., \& Musson, G. (2002). When 'Work' Meets 'Home': Temporal flexibility as lived $\begin{array}{lllll}\text { experience. } & \text { Time }\end{array}$ 
International Journal of Business Management and Economic Review

Vol. 4, No. 04; 2021

ISSN: 2581-4664

https://doi.org/10.1177/0961463X02011002008

Tiroina, S. De, \& Mahdani, S. (2021). The Effect of Work From Home during the COVID19 Pandemic on Work-life Balance and its Impact on Employee Performance of Aceh Communication, Informatics and Encoding Office, 4(02), 55-63.

Vagias, \& Wade, M. (2006). Likert-type scale response anchors. Clemson International Institute for Tourism and ..., 3-4. https://doi.org/10.1525/auk.2008.125.1.225 